\title{
Possible Source of the Gompertz Law of Proliferating Cancer Cells: Mechanistic Modeling of Tumor Growth
}

\author{
K.W. Fornalski ${ }^{a, b, *}$, J. ResZCZYŃSKa ${ }^{a, c}$, \\ L. DoBrzyŃski ${ }^{a}, \mathrm{P}$. WYSOCKI ${ }^{d}$ AND M.K. JANIAK ${ }^{e}$ \\ ${ }^{a}$ National Centre for Nuclear Research (NCBJ), A. Soltana 7, 05-400 Otwock-Świerk, Poland \\ ${ }^{b}$ Ex-Polon Lab, Podleśna 81a, 05-552 Eazy, Poland \\ ${ }^{c}$ Medical University of Warsaw (WUM), Department of Biophysics and Human Physiology, \\ Chałubińskiego 5, 02-004 Warszawa, Poland \\ ${ }^{d}$ Faculty of Physics, Warsaw University of Technology, Koszykowa 75, 00-662 Warszawa, Poland \\ ${ }^{e}$ Military Institute of Hygiene and Epidemiology (WIHE), \\ Kozielska 4, 01-163 Warszawa, Poland
}

Received: 13.07.2020 \& Accepted: 10.08.2020

Doi: 10.12693/APhysPolA.138.854

*e-mail: krzysztof.fornalski@ncbj.gov.pl

\begin{abstract}
Induction and proliferation of cancer cells are complex processes whose $a b$ initio mathematical description is virtually impossible. Nevertheless, some integral characteristics such as the Gompertz law, which is generally used to describe cancer development, can result from a simple mathematical consideration of biophysical processes. The simplified response to a single-source lesion created in a cell's DNA consists in the repair of the lesion or induction of a mutation which can lead to neoplastic transformation. This approach makes it possible to propose a single mathematical formula connecting the Gompertz curve with the simplified biophysical characteristics of tumor growth. Such an approach, which merges the process of neoplastic transformation with cancer development, is shown in the present paper based on the relatively simple analytical and Monte-Carlo models. The models operate with a small number of biophysical parameters and account, at least in part, for the mechanisms that operate in an organism exposed to low doses of ionizing radiation.
\end{abstract}

topics: cancer biophysics, Gompertz curve, ionizing radiation, cancer cell dynamics

\section{Introduction}

Historically, the phenomenological relationship of the cancer cells' dynamics is usually described by the Gompertz curve [1] which displays the nonsymmetrical sigmoidal growth. This function starts gradually, then, after a short time, becomes quasiparabolic and is followed by a rapid growth. The progression phase of a tumor exemplifies this type of dynamic behavior. With time, the number of cancer cells (i.e., the volume of the tumor) saturates very slowly to a limiting constant value. This is commonly explained by the shortage of nutrients necessary to maintain the tumor growth. All of this can be described exactly by the Gompertz law - still not fully understood - which presents the phenomenological approximation appearing to be the most accurate. In fact, it can be derived from the phenomenological differential equation, similarly to many other possible functions exhibiting a sigmoidal shape [2].

Since the first application of the Gompertz curve to real experimental data [1], scientists all over the world have created many models which tried to explain it in the context of the carcinogenesis process. First, the paper by Bozic et al. [3] should be quoted. They maintained that the development of tumors starts from a single driver mutation and modeled the development of "passenger" mutations in a tumor as a function of the driver mutations. In contrast to that, Little and Hendry [4] intended to separate two processes: the process when the mutation and the development of cancer are the result of a metabolic process and the process in which the situation is changed by a specific mutagen, such as ionizing radiation or cigarette smoking. Considering the two scenarios, at the end, the results were dramatically different (depending on the parameter used, the cancer risk could change even by 20 orders of magnitude). The problem of what happens before the tumor starts forming was disscussed in [5]: driver and passenger mutations are modeled.

From the practical application's point of view, Schneider [6] has created a mechanistic model of the secondary carcinogenesis after fractionated radiotherapy. Shuryak et al. [7] have merged and developed many existing models which were divided into two groups: (i) long-term models "that track 
premalignant cell numbers throughout an entire lifetime but treat initial radiation dose-response simplistically", and (ii) short-term models "that provide a detailed initial dose-response (...) but address its modulation during the subsequent cancer latency period only indirectly" [7]. This approach seems to be very deep and complex, and generally meets most of experimental findings. However, the long-term tumor growth is not a trivial thing which was additionally proved by Khordad and Rastegar Sedehi [8] who modeled cancer dynamics with the use of entropy and statistical mechanics both typically used in physics. Of course, one can find much simpler modeling approaches which can be useful for some general applications [9] or mechanistic models focused on nonlinear aspects of low doses of ionizing radiation [10].

The present paper offers an analytical foundation of the Gompertz law in the context of the process of carcinogenesis that starts with the creation of a DNA damage which, when unrepaired, may produce a fixed mutation and lead to a neoplastic transformation of a cell. The simple analytical formula describing the transition from the initiating event to the clinically detectable tumor is presented and discussed within its applicability. It turns out that the Monte Carlo simulation also shows that many different biophysical processes taken into account in the simulation yield the Gompertzian relationship of the kinetics of the created cancer cells.

Our paper proposes a model of cancer cell creation and growth different from many models widely presented in the scientific literature (see above) and shows that basic biophysical laws with some simplifications are sufficient to explain the Gompertzian shape of the cancer dynamics.

\section{DNA damage in a single cell}

Let us denote the probability density function (PDF) of the creation of a DNA damage in a single cell as $P_{L}$. The exact form of $P_{L}$ represents the way how a detrimental agent affects the DNA. In the general case of many different and independent agents, one can present this PDF as a sum of separate processes

$$
P_{L}=\frac{\sum_{a=1}^{A} w_{a} P_{L, a}}{\sum_{a=1}^{A} w_{a}},
$$

where $A$ denotes the maximal possible number of all different detrimental agents which can affect the DNA and $w_{a}$ denotes the weight with which a given agent participates in the whole process. When no external agents are present, the DNA can be affected by a chemical reaction with metabolic reactive oxygen species (ROS, mostly radicals) as well as by erroneous DNA replication. The PDF of that process can be given by the equation of the ROS diffusion, analogically to the transfer of detrimental signals from the targeted to bystander cells [11]. In the first approximation, it can be, however, treated as going with a constant rate.

In the case of external agents, their interaction with the DNA can be twofold: a general homogeneous interaction (as in the case of chemical agents or ROS generated by external agents) and a single hit instantaneous interaction (as in the case of a direct hit by ionizing radiation). In the former case, one can assume a linear dependence related to the homogeneous concentration $(\rho)$ of the agent(s) in a cell

$$
P_{L, \text { hom }}=\sum_{i=1}^{n} \alpha_{i} \rho_{i},
$$

where, in contrast to $A, n$ denotes the number of external agents only and $\alpha_{i}$ denotes the appropriate weights.

The concentration of ROS from radiolysis (mainly $\mathrm{O}_{2}^{\bullet-}, \bullet \mathrm{OH}$ ) and $\mathrm{H}_{2} \mathrm{O}_{2}$ depends on the linear energy transfer (LET) and the dose of radiation as well as on a cell type. The total number of oxidative hits to the DNA is estimated to be about $10^{9}$ per cell per day [12]. The spectrum of the radiationgenerated ROS is similar to that formed during metabolic processes with one major difference: the damage from metabolic ROS is randomly distributed in the DNA, while the radiation-induced DNA damage frequently occurs in clusters [13].

In the case of direct hits, one can use the PDF formula proposed by L. Dobrzyński et al. [14], analogical to the target theory, for a special case of the ionizing radiation interaction as

$$
P_{L, \text { hit }}=C \sigma\left(1-\mathrm{e}^{-a D}\right),
$$

where $D$ denotes the radiation dose, $C$ is the scaling constant and $\sigma$ is the physical macroscopic cross-section of the radiation interaction with matter. We believe that the simplifications used so far should provide a sufficient description of the reality.

\section{Mutation induction}

The creation of an oncogenic mutation results from the unrepaired or improperly repaired alteration of the nucleotide sequence of the coding part of the DNA. Hence, such a mutation is strictly connected with $P_{L}$ and $P_{R}$, the latter denoting the PDF of all possible repair mechanisms. The value of $P_{L}\left(1-P_{R}\right)$ represents the PDF of the DNA alteration which was not repaired (or improperly repaired) only. This is, however, not sufficient for the induction of an oncogenic mutation. One needs to introduce an additional term based on different and independent mechanisms of the induction of such a mutation in a single cell with a single unrepaired alteration (lesion):

$$
P_{\text {ind }}=\frac{\sum_{i} w_{i} P_{\text {ind }, i}}{\sum_{i} w_{i}},
$$


where $w_{i}$ denotes the weight of lesion from a given $i$-th cause. The exemplary $P_{\text {ind, } i}$ function can be found in the German model called the random coincidence model-radiation adapted (RCM-RA) [15]. Another example, but limited to radiation only, can be deduced from the dual radiation action model [16]. This specific case is limited to chromosomal aberrations only which are the example of the radiation-induced double-strand break's oncogenic mutations - the most frequently considered in experimental radiobiology and cytogenetics. The presented approach will continue this line of reasoning as the most mathematically described case which is linear or linear-quadratic at low doses and saturates at very high doses [17]:

$$
P_{\text {ind, rad }}=1-\exp \left(-\sum_{R} \sum_{i=0} a_{i, R} D_{R}^{i}\right),
$$

where $R$ represents the radiation type (i.e., high or low LET) and $a_{i, R}$ 's are the experimentally derived constants. For ionizing radiation only, $D$ represents the radiation dose. As a matter of fact, (5) can be used as a more universal one and in this context $D$ can be replaced by any analyzed agent $\xi$. Now, let us assume that $P_{\text {ind }} \approx P_{\text {ind, } \operatorname{rad}}(\xi)$.

Finally, the PDF of the induced mutation can be presented in the most general form as

$$
P_{M}=P_{L} P_{\text {ind }}\left(1-P_{R}\right),
$$

where the PDFs are given by $(3)-(5)$. It is also possible to introduce any other form which will be appropriate for a given type of a detrimental agent [14, 17].

Additional comments should be made regarding the repair of a DNA lesion $P_{R}$. Such a repair can be regarded as a natural $P_{N R}$ and/or adaptive response $P_{A R}$ to a toxic agent. This approach was described in [14] and its formalism will be used in further considerations. One should remember, however, when considering the fate of a lesioned cell, that a repair process may restore its healthy state (described in detail in Sect. 6).

Let us stress that in our mathematical treatment we avoid detailed, microscopic biological processes and the description bears integral characteristic only.

Figure 1 shows an exemplary shape of the $P_{M}$ function related to the dose $D$ of ionizing radiation. The black solid curve corresponds to the situation in which $P_{R}=P_{N R}=$ const, while the gray curve corresponds to $P_{R}=P_{N R}+P_{A R}(D) \neq$ const. For $P_{A R}(D)$, the function identical to that employed in [14] was used. It can be observed that within a certain range of doses the value of $P_{M}$ due to a radiation-induced adaptive response $\left(P_{A R}>0\right)$ is lower than the value of $P_{M}$ without that effect. When potent repair mechanisms are activated, the so-called hormetic effect appears - a local minimum in $P_{M}(D)$ function. When the repair mechanisms are arbitrarily turned off $\left(P_{A R}=0\right)$ or the dose is too high, the relationship $P_{M}(D)$

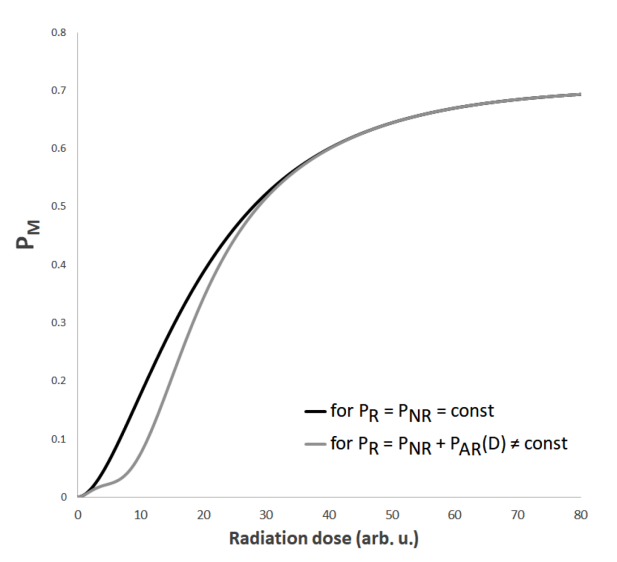

Fig. 1. Exemplary shapes of $P_{M}$ given by (6) regarding ionizing radiation as a detrimental agent, including the probability of the repair processes $\left(P_{R}=\right.$ const for the black curve and $P_{R} \neq$ const for the gray curve associated with the radiationinduced adaptive response). Noticeably, the difference between both curves represents the beneficial (hormetic) effect of irradiation.

merges with the black line. These findings are consistent with those of [17], where the probability of a fixed mutation is very similar to the one given by (6). It was assumed, for simplicity, that internal (metabolic) detrimental agents are absent in Fig. 1 (hence, both curves in the figure start from zero, $\left.P_{M}(D=0) \approx 0\right)$. This does not happen in reality where additional mutations can occur $\left(P_{M}(D=0)>0\right)$. Consistently, when the repair mechanisms eliminate both radiation- and nonradiation-induced lesions, the curves in Fig. 1 may assume a hormetic shape.

\section{Neoplastic transformation}

Within the framework of the presented model, the probability of neoplastic (i.e., cancer) transformation of the affected cell is strictly related to the number of oncogenic mutations $m$ accumulated in the DNA. According to the current understanding, the process of carcinogenesis (i.e., the creation of a cancer) is regulated by two main groups of the so-called driver genes. The first group consists of proto-oncogenes which, upon a "gain-of-function" mutation, behave as oncogenes and contribute to the neoplastic transformation of a mutated cell. Genes of the second group are called tumor suppressor genes whose "loss-of-function" mutation creates the danger of losing cells' self-control [18]. Out of the total number of about 30,000 genes in the human genome, $\pi=299$ have been identified as driver genes [19].

In this context, it is important to note that the RCM-RA model of Fleck et al. [15], which is based on Moolgavkar's multistage theory [20], describes the process of mutation accumulation in relation to the probability of a single cell mutation $\mathrm{P}_{M}$ [14]. Thus, based on this model, 
the time-dependent generation of $\mathrm{M}_{m}$ cells with one oncogenic mutation $\left(\mathrm{M}_{1}\right)$ can be described as

$$
\frac{\mathrm{d} M_{1}}{\mathrm{~d} t}=\left(M_{0}-M_{1}\right) B P_{M},
$$

where $M_{0}$ denotes the number of non-mutated cells and $B$ represents the factor related to the number of cancer-critical genes. This factor, however, is time dependent and is related to the number of mutations $B(t, m)$. Usually, when no additional cancerogenic hazards exist, $B$ is measured in years $^{-1}$ regarding the entire human being [20]. Additionally, the number of mutations is much smaller than the number of oncogenes in non-neoplastically transformed cell $(m \ll \pi)$. Therefore, one can assume that $B$ is generally constant regardless of the number of the existing mutations [14]. Summing up information presented about the number of driver genes $\pi$ with respect to the total number of genes [19], one can estimate the coefficient $B$, which describes the critical potentially carcinogenic region in the DNA, to be not higher than 0.01 per year.

Note also that almost an identical differential equation can be written for the dose dependence. In such a case, the differentiation with respect to time is substituted obviously by the differentiation with respect to a dose and the value of $B P_{M}$ has to be substituted by another constant. Then, solution of (7) is

$$
M_{1}=M_{0}\left(1-\mathrm{e}^{-B P_{M} t}\right) .
$$

Analogically, one can find the solution for the number of cells with $m$ mutations in cancer-critical genes before neoplastic transformation [14]:

$$
M_{m}=M_{0}\left(1-\mathrm{e}^{-B P_{M} t}\right)^{m} .
$$

Generally, the experimental data show that a certain number of the driver gene mutations $m \in[2,8]$ is required for a mutated cell to transform into a cancerous one [21-24]. Moreover, the probability of this transformation increases very rapidly around $m_{\text {average }} \approx 5$. This relationship can be presented phenomenologically as a sigmoidal curve. The shape can be obtained also by the AvramiMehl type of equation [14, 17] — originally derived for the description of the kinetics of a phase transition when a new phase is formed within another one. Thus,

$$
P_{A-M}=1-\exp \left(-c m^{k}\right),
$$

where $k$ is the critical index, which in the description of the kinetics of tumor growth is associated with the dimensionality of this growth, and $c \approx 0.0277$ when $k=2[14]$. In the original Avrami's approach, one considers that the growth rate of the new phase is an exponential function of time $\tau$, proportional to $\tau^{k}$. Therefore, taking full advantage of this theory, it is tacitly assumed that the number of mutations increases proportionally to the characteristic time in which a cancer transformation takes place. In other words, in such an approach one uses two time scales: one for a formation of mutations and another one for a transformation of a mutated cell to the cancer cell [17].

\section{Tumor growth}

Assuming that $m$ mutations are required for a cancerous transformation of a cell, one can write the formula for that transformation for all $M_{m}$ cells (and the further growth of the whole tumor) as

$$
P_{C}(m)=M_{m}(t, m) P_{A-M}(m),
$$

which can be presented in a complete form for a given number of mutations as in [14]:

$$
P_{C}(m)=M_{0}\left(1-\mathrm{e}^{-c m^{k}}\right)\left(1-\mathrm{e}^{-B P_{M} t}\right)^{m}
$$

and $P_{M}$ is assumed to be time-independent for fixed values of input parameters. Therefore, (12) can be used as a cancer cells generator.

In order to obtain the full probability of the development of a cancer, one should sum up (12) over all possible values of $m$. In other words: $P_{C, \text { tot }}=\sum_{m} P_{C}(m)$ [14] which is also consistent with the multistage theory of carcinogenesis [20]. One can additionally use the time shift $t \rightarrow\left(t-t_{0}\right)$, where $t_{0}$ denotes the minimum time when cancer cells may appear. The final form of the probability function for the cancer cells growth with an initial neoplastic transformation can be then presented as

$$
\begin{gathered}
P_{C, \text { tot }}=\sum_{m=1}^{10} M_{0}\left(1-\mathrm{e}^{-c m^{k}}\right) \\
\times\left(1-\mathrm{e}^{-B P_{M}\left(t-t_{0}\right)}\right)^{m},
\end{gathered}
$$

where some constant values $c \approx 0.0277$ and $B \leq 0.01$ year $^{-1}$ [14], while $P_{M}$ is defined by (6). Some additional information needs to be presented regarding the parameter $k$.

Figure 2 shows to what extent the final results are dependent on the assumed values of the critical index $k$. The slope of the curve may vary in the two exemplary cases $(k=2$ and $k=4)$, resulting

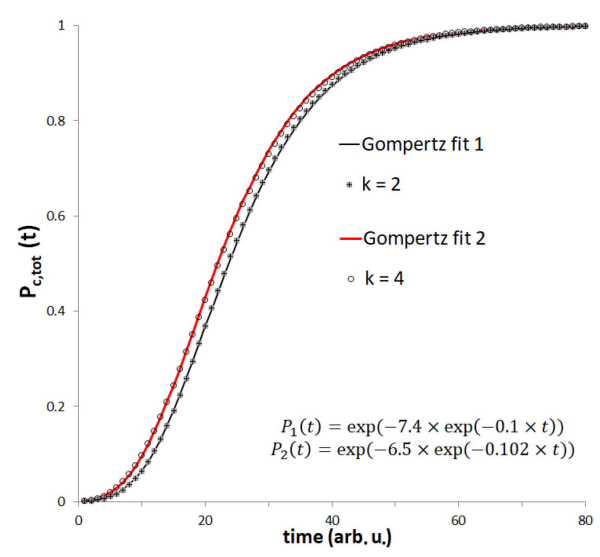

Fig. 2. Probability $P_{C \text {, tot }}$ of a neoplastic transformation vs. time assuming two values of the critical index, $k=2$ and 4 , for comparison. The formula (12) was summed over $m$ from 1 to 10 [14] to simulate exemplary data point (filled and empty circles). Solid curves are fits of the normalized Gompertz relationships (14) presented as $P_{1}(t)$ (lower curve for $k=2$ ) and $P_{2}(t)$ (upper curve for $k=4$ ). 

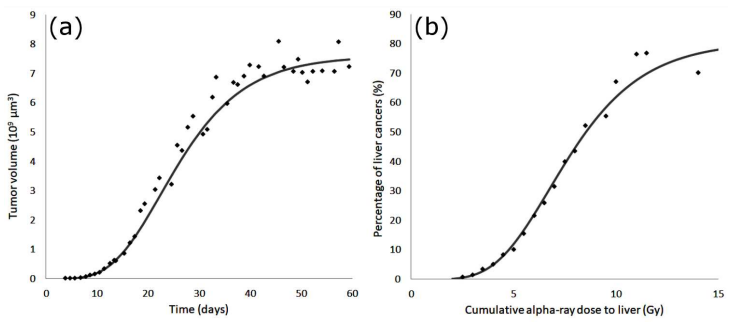

Fig. 3. The application of (13) to some exemplary experimental data: (a) time-related growth of fibroblast cells, where $M_{0}=1.45, k=2$, $B P_{M}=0.113$ and $t_{0}=3.5$; original data taken from Fig. 4 in [2]; (b) dose-related increase of liver cancers among Danish patients exposed to thorotrast, where $M_{0}=15.43, k=2, B P_{M}=0.408$ and $D_{0}=1.5$; original data taken from [25].

in the pure Gompertz curve which well explains the data. Such a situation can most likely be related to the fact that the first term in (13), independent of time, is nothing else than the weight of the last term and it averages out after the summation. Hence, the final shape of the curve is dependent mainly on the last term in (13) which is time-dependent. It follows from Fig. 2 that the pure Gompertz curve can perfectly approximate the mechanistic formula given by (13).

Therefore, (13) arises as a novel mechanistic model of cancer growth which can be easily verified on the real experimental data. A good example to refer to is Fig. 4 in the paper by Marušić et al. [2], where fibroblast cells (line V79) were growing spherically according to the sigmoidal shape of their dynamics. The result of fitting the model given by (13) is presented in Fig. 3a.

The proposed approach is much wider. When the time is going towards the dose, namely $t \rightarrow D$ and $t_{0} \rightarrow D_{0}$, the character and applicability of the curve remain the same (only the constant in front of the dose changes). This is presented in Fig. 3b where the modified (13) was fitted to the liver cancer cases in Denmark [25]. One should note that the data in Fig. $3 \mathrm{~b}$ are by no means belonging to a low-dose regime.

\section{Tumor development and the Gompertz law}

Let us examine the last term on the r.h.s. of (12) and analyze to what extent it may be identified with the Gompertz curves presented in Fig. 2.

Let us recall the argument of the function $g(t)=(1-\exp (-\beta t))^{m}$, where $\beta \equiv B P_{M}$. As mentioned earlier, parameter $B$ corresponds to the ratio of the number of critical (i.e., carcinogenesisrelated) genes to the number of all genes, thus $B<1$ per year. Additionally, $P_{M}$ can be maximally equal to 1 , so the condition $\beta=B P_{M}<1$ per year is always true. For practical purposes, the $\beta$ value in Fig. 2 was set to 0.1 [14] and it is equivalent in the calculations to a decrease of the time unit by 10 . Consistently, $\beta t$ in $g(t)$ varies from zero to 10 for all the values of $m$.

The Gompertz curve is typically described by:

$$
f(t)=\exp \left(-a \mathrm{e}^{-\beta t}\right)
$$

and differs from the one presented by (12), reduced to the function $g(t)$. However, when one looks at differential equations fulfilled by both functions

$$
\left\{\begin{array}{l}
\frac{\mathrm{d} f}{\mathrm{~d} t}=a f \beta \exp (-\beta t), \\
\frac{\mathrm{d} g}{\mathrm{~d} t}=g m \beta \exp (-\beta t)[1-\exp (-\beta t)]^{-1},
\end{array}\right.
$$

it becomes clear that for $\beta t \gg 1$, when $\mathrm{e}^{-\beta t} \ll 1$, both functions have the same underlying differential equations. Furthermore, let us consider the limiting relationships, i.e.,

$f(t)= \begin{cases}\mathrm{e}^{-a \mathrm{e}^{-\beta t}} \approx\left(1-\mathrm{e}^{-\beta t}\right)^{a} \cong 1 & \text { for } \beta t \gg 1, \\ (1+a \beta t) \mathrm{e}^{-a} & \text { for } \beta t \ll 1,\end{cases}$

and

$$
g(t)=\left\{\begin{array}{ll}
\left(1-\mathrm{e}^{-\beta t}\right)^{m} \cong 1, & \text { for } \beta t \gg 1 \\
(\beta t)^{m}, & \text { for } \beta t \ll 1
\end{array} .\right.
$$

As noted in (16) and (17), it is clear that $\lim _{t \rightarrow \infty} f(t)=\lim _{t \rightarrow \infty} g(t)=1$ which is equivalent to the saturation of both sigmoidal curves at very long times.

This is just another way to see that the biggest difference between these two functions occurs at low $\beta t$ values. However, if $\beta t$ is large, the function $\left(1-\mathrm{e}^{-\beta t}\right)^{a}$ is identical to $g(t)$ when $a=m$. On the other hand, to get the full probability of development of cancer, one has to sum up (13) over $m$. Hence, from (17) one obtains

$$
\sum_{m=1}^{10}(\beta t)^{m} \approx \frac{1}{1-\beta t}-1 \approx \beta t \quad \text { for } \beta t \ll 1 .
$$

The main weakness of using the Gompertz function as $f(t)$ is that it does not start from zero at $t=0$. This indicates that the development of cancer in its very initial stage cannot be described with the Gompertz curve mainly because - in its original assumption - it starts from the non-zero cancer volume [1]. At longer times, the problem disappears which simply confirms that the results presented in Fig. 2 are well understood. In fact, solid tumors are currently detectable clinically when they are sufficiently large, i.e., a relatively long time after the initiation phase of a carcinogenesis.

\section{Monte Carlo simulations}

A group of cells can be modeled using the memory Markov chain Monte Carlo (MMCMC) method where practically all the important but simplified biophysical mechanisms can be taken into consideration in the form of a probability tree [26-28]. The simulations performed in this study took into account the impact of ionizing radiation only and disregarded other external detrimental agents. With the MMCMC method, one can present 


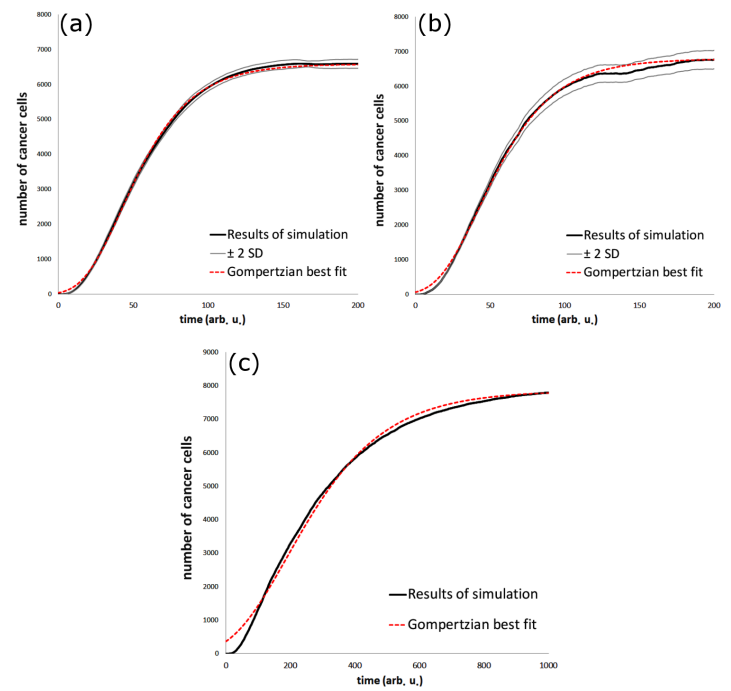

Fig. 4. Exemplary results of the Monte Carlo simulation of the dynamics of the creation of cancer cells which support the Gompertzian shape for: (a) a full tree of probabilities, (b) a narrowed tree of probabilities, (c) a strongly narrowed tree of probabilities (the Gompertzian shape is of lesser use, especially at the lowest times). Red dashed curves correspond to the Gompertzian best fit, black solid curves represent results of the simulation, while gray curves represent the simulation uncertainty (two standard deviations, SD; except (c) where uncertainties were not significant). The constant irradiation (dose-rate) was implemented.

an additional proof that the Gompertz curve appears in a natural way when the basic biophysical effects are taken into account.

The simulation was based on the assumption that each cell remains in one of the four possible states

- healthy,

- damaged,

- mutated,

- cancerous.

Each state is represented by the proper probability functions located on the broad tree of probabilities. One has to note, however, that in this paper the irradiation of cells is treated in a purely mechanistic way and no specific microdosimetric Monte Carlo calculations of DNA damages are carried out. Such calculations can be found in [29,30].

The simulations were carried out under the following conditions: the initial number of cells (cluster size) was set at 9000; all of the cells are healthy; the number of the Monte Carlo time steps has been limited to 800. The parameters necessary for the description were estimated or taken ad hoc [28].

The overall simulation which takes into account all (or most of) the biophysical mechanisms of irradiated cells shows that the number of cancer cells appearing with time is well described by the Gompertz relationship (see Fig. 4a). It also turns out that the logarithm of the survival of cells is described by the linear-quadratic relationship as commonly accepted. The compatibility of the modeled points with the Gompertz function is very high $\left(R^{2}=0.999\right)$. A valid question is which branches of the probability tree decide on this character. Alternatively, one could find which of the branches are irrelevant for arriving at the Gompertz-type behavior.

All biological effects of our interest were described in previous studies [26-28] and they will not be repeated here. Admittedly, an analytical solution which would include all the analyzed biological processes is rather impossible. In particular, the analysis of the bystander effect requires $3 \mathrm{D}$ simulations of the cell-cell interactions. Surprisingly, the shape of the Gompertz function survives (Fig. 4b) when the number of branches in the probability tree is strongly reduced and calculations are uniquely limited to the following mechanisms:

- for a healthy cell — to the radiation-induced lesion;

- for a lesioned cell - to the induction of a mutation or an additional lesion after exposure to radiation; alternatively, to the induction of a spontaneous mutation not due to a radiation hit;

- for a mutated cell - to its neoplastic transformation after a radiation hit;

- for a cancer cell - to all the mechanisms induced in an irradiated and non-irradiated cancer cell, namely: spontaneous death, multiplication, bystander effect, death due to a precise radiation hit, etc.

The result of the MMCMC simulation for this narrowed tree is presented in Fig. 4b which is not much different from the one shown in Fig. 4a. Indeed, the Gompertz function fits here very well.

In the next step, a still narrower path for each cell's state was allowed. Namely:

- for a healthy cell — only the probabilities of the radiation hit to the DNA $\left(P_{\text {hit }}\right)$ and of the lesion induced by such a hit $\left(P_{R L}\right)$; thus the probability of the creation of a lesion in a healthy cell is reduced to the product $p_{H \rightarrow L}=P_{\text {hit }} P_{R L}$;

- for a lesioned cell — since a radiation hit to the DNA $\left(P_{\text {hit }}\right)$ can produce a mutation with probability $P_{R M}$, one can rewrite the probability of the creation of the post-irradiation mutation as $p_{L \rightarrow M}=P_{\text {hit }} P_{R M}$;

- for a mutated cell - since after the mutationinducing DNA hit $\left(P_{\text {hit }}\right)$ the cell can undergo neoplastic transformation $\left(P_{R C}\right)$, thus $p_{M \rightarrow C}=P_{\text {hit }} P_{R C}$;

- for a cancer cell - no action.

The probability functions presented above are listed in analytical forms in Table I. An exemplary result of the simulation carried out under the aforementioned conditions is shown in Fig. 4c which depicts 
TABLE I

Selected formulae of the probability functions used in the Monte Carlo modeling (see also [26-28]). Remarks: $D$ - ionizing radiation single dose, $P_{\text {hit }} \neq P_{R L}, L-$ number of lesions in the cell's DNA, $m$ - number of mutations, $k$ - critical index

\begin{tabular}{|c|c|}
\hline $\begin{array}{c}\text { Description } \\
\text { of the process }\end{array}$ & $\begin{array}{c}\text { Probability } \\
\text { function formula }\end{array}$ \\
\hline DNA hit by radiation $^{a}$ & $P_{\text {hit }}=1-\mathrm{e}^{- \text {const } D}$ \\
\hline $\begin{array}{l}\text { DNA lesion creation } \\
\text { by a radiation hit }\end{array}$ & $P_{R L}=1-\mathrm{e}^{-\operatorname{const} D}$ \\
\hline $\begin{array}{l}\text { mutation creation } \\
\text { from the lesion(s) }\end{array}$ & $P_{R M}=1-\mathrm{e}^{- \text {const } L}$ \\
\hline $\begin{array}{l}\text { cancer transformation } \\
\text { of the mutated cell }\end{array}$ & $P_{R C}=1-\mathrm{e}^{- \text {const } m^{k}}$ \\
\hline \multicolumn{2}{|c|}{$\begin{array}{l}{ }^{a} \text { see Appendix B in [14] for details of the formula } \\
\text { derivation; } \\
{ }^{b} \text { see analogous (10) based on the Avrami-Mehl } \\
\text { equation }[14,17] .\end{array}$} \\
\hline
\end{tabular}

the closest situation to the one given by (13). Noticeably, the Gompertz curve fits quite compatible with the simulated data - with a lower compatibility but still on an acceptable level $\left(R^{2}=0.995\right)$. Additionally, the simulated curve covers a broader range of times (see the time scale change in Fig. 4c). A simple inspection of Fig. 4c reveals that, for this case, the proposed tree seems to be oversimplified to reflect the Gompertz shape well, especially at low values of time. However, in contrast to the two previously discussed cases, the latter case can be easily presented also in the analytical way which is an important advantage.

Obviously, the Monte Carlo approach presented in this paper is a numerical simulation. However, due to the narrowing of the original probability tree, it is possible to describe the time growth of cancer cells analytically, using the probabilities listed in Table I.

\section{Analytical solutions for the Monte Carlo simulations}

Within the scope of this model, an increase in the number of the lesioned cells with time can be described as

$$
\begin{aligned}
& L(t)=C_{H \rightarrow L} p_{H \rightarrow L} t= \\
& \quad C_{H \rightarrow L}\left(1-\mathrm{e}^{-c_{1} D}\right)\left(1-\mathrm{e}^{-c_{2} D}\right) t,
\end{aligned}
$$

where $C_{H \rightarrow L}=$ const is a conversion factor needed for the transformation of the probability value to the number of cells.

Analogically, the number of the mutated cells can be presented as

$$
\begin{aligned}
& m(t)=C_{L \rightarrow M} p_{L \rightarrow M}= \\
& C_{L \rightarrow M}\left(1-\mathrm{e}^{-c_{1} D}\right)\left(1-\mathrm{e}^{-c_{3} L(t)}\right)
\end{aligned}
$$

and the number of emerging cancer cells as

$$
\begin{aligned}
& N(t)=C_{M \rightarrow C} p_{M \rightarrow C}= \\
& \quad C_{M \rightarrow C}\left(1-\mathrm{e}^{-c_{1} D}\right)\left(1-\mathrm{e}^{-c_{4} m(t)^{k}}\right) .
\end{aligned}
$$

Inserting (19) into (20) and merging the result with (21), one can arrive at the sigmoidal function (similar to the Armitage-Doll model [31]) for the increase in the number of cancer cells with time

$$
N(t)=\tilde{C}_{1}\left[1-\exp \left(-\tilde{C}_{2}\left(1-\mathrm{e}^{-\tilde{C}_{3} t}\right)^{k}\right)\right]
$$

assuming that the dose used in the calculations is time-independent (which can be treated as a dose-rate or a single radiation pulse) and, consequently, all the dose-related functions are also time-independent. As mentioned earlier, the narrowed tree of probabilities, here represented analytically by (22), does not produce a perfectly Gompertzian curve (Fig. 4c) but this shape serves as quite a good approximation of the exact formula. Moreover, its simplified form can be very useful for further theoretical considerations, especially for the analysis of different behaviors of cancer cells (see the next section).

The simplified approach presented above may be carried out both analytically or numerically. However, the analytical result obtained using (22) is practically the same as in its Monte Carlo ana$\log$ in Fig. 4c. In the particular case described in this section, both approaches can be treated as equivalent.

\section{Cancer cells interactions}

As mentioned in the previous section, (22) was derived assuming that no changes in the number of the existing cancer cells are expected. Typically, however, cancer cells multiply (with the constant probability $P_{C M}$ ), naturally die off (constant probability $P_{C N D}$ ) or are killed by a potent radiation hit or due to their inherent hyper-radiosensitivity (time-independent probabilities $P_{C R D 1}$ and $P_{C R D 2}$, respectively, given by the formulae analogous to $P_{R L}$, see Table I) [26-28]. The multiplication and natural deaths of the cells can occur irrespectively of irradiation, however, $P_{C R D}=P_{C R D 1}+P_{C R D 2}$ can be taken into consideration prior to the radiation hit only, so $P_{\text {death }}=P_{\text {hit }} P_{C R D}$.

Such a reasoning can be readily applied to the analytical solution of the narrowed tree of probability from the previous section. Using the analogous approach as in (19)-(21), one can calculate the correction factor

$$
\Delta N(t)=t\left(P_{C M}-P_{C N D}-\tilde{C}_{4} P_{C R D}\right)
$$

which should simply modify (22) to obtain its more general formula for the cancer cells' dynamics which includes different cellular behaviors

$$
\tilde{N}=(1+\Delta N) N \text {, }
$$

where $N$ is given by (22) and $\Delta N$ by (23). This modification can influence the Gompertzian shape if $|\Delta N|$ is large enough. 


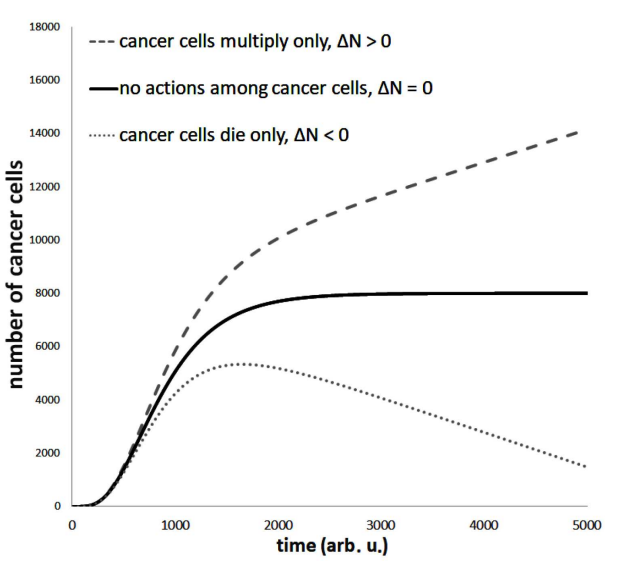

Fig. 5. Exemplary analytical calculations of the total number of cancer cells vs. time corresponding to different behaviors of cancer cells: multiplication only (upper dashed line), death only (lower dotted line) or no changes in the number of already created cells (black middle line); this last case is analogous to Fig. 4c. The calculations were carried out using (24).

Many experimental data (e.g., [32]) usually present the Gompertzian shape of $N(t)$, so, in most cases, $\Delta N \approx 0$. This means that $(23)$ can be viewed as a balance equation when multiplication (proliferation) of the cells competes with their mortality. However, in some cases tumor cells multiply very rapidly $(\Delta N>0)$ which distorts the Gompertzian shape. The most famous example are the immortal HeLa cells which can multiply infinitely in culture. The opposite situation is when cancer cells are injected into the body where, if the cells are genetically incompatible with the host, they die off pretty fast. In any case, all those behaviors can be described by (24) with the Gompertz curve as the most typical response $(\Delta N \approx 0)$, other solutions also being possible $(|\Delta N|>0)$ (see Fig. 5). This means that the Gompertz curve describes the most typical case among the broad family of the cancerdynamics' functions [8].

\section{Discussion}

Experimental examinations of the growth (proliferation) of cancer cells have been available for decades [1, 2, 32]. Examples of interesting modeling of initiation and development of cancers can also be found in many papers $[3-5,7,10]$. However, the dose-effect curve has rarely been discussed. The Gompertz law, which is now a golden standard in medical physics and radiobiology, was examined in many ways, although no detailed analysis of its relation to crucial physical processes of carcinogenesis has been demonstrated so far. Hence, the present paper provides a simplified biophysical explanation of such an integral characteristics of the growth of cancer cell like the Gompertz law which was often found experimentally but has not been satisfactorily explained so far.
Apparently, the Gompertz behaviour does not seem to arise from the particular shape of the $P_{M}$ function (Fig. 1). In fact, such a shape may follow from the exact number of mutations (in the function of time) but how these mutations were created is less important during the final stage of neoplastic transformation (Fig. 2). When $P_{M}$ is given by a different function (related to some other detrimental agents), the final Gompertzian shape of (12) and (22) remains but some geometrical shifts along the $x$-axis can be observed due to the differences in the complexity of the creation of oncogenic mutations.

It is important to note that the analytical origin of the Gompertz law was supported by the Monte Carlo simulation (Fig. 4a) which operates on probabilities of different simplified biological effects. Indeed, such a simulation appears to be very useful because the user can cut out or add particular branches to the probability tree (Fig. 4b). As a result, the pure path to the Gompertz shape can be analyzed separately which was presented in Fig. 4c with an additional analytical description for this case.

Figure 4c was created under the assumption that cancer cells are passive after their creation. Thus, the tumor growth presented in Fig. 4c is associated with the appearance of new cancer cells due to the neoplastic transformation of the mutated cells only. However, when additional probabilities associated with the existing cancer cells (e.g., rates of their proliferation, death, etc.) are implemented, the Gompertzian shape can be modified - the constant saturation for a long period of time (Fig. 4) can be replaced by a tendency to approach zero or infinity, as presented in Fig. 5. This suggests that the Gompertz curve is just a special case of a much wider family of the time-response curves describing the dynamics of cancer cells.

Our most important general finding is that the Gompertzian shape of the cancer cells' dynamics was obtained independently by two different approaches, namely analytical calculations of the purely mathematical transition from a single DNA lesion to a neoplastic transformation and the analogous Monte Carlo simulation. Eventually, these two different analyses yielded the same outcome. Hence, we can conclude that: (i) the calculations are, in general, correct, (ii) the general way of thinking about the biophysics of the development of cancer is correct, and (iii) the Gompertz curve naturally follows from the cancer cells' standard behavior at longer times. One has to remark, however, that the difference between various shapes (Gompertzian, Avrami's, logistic, more or less detailed simulations and our mechanistic approach) is very minute at long times. This means that the agreement of the experimental data with a given sigmoidal shape can hardly be used as a proof of the correctness of the phenomenological model used. 
Finally, as indicated in Fig. 4 for the small time scale, we may suggest that the Gompertzian shape is rather inappropriate for the description of the early phase of the tumor growth. This is also supported by (17) and it is likely that a parabolic function can be more appropriate in this case. Clinically, however, the very early phase of tumor growth is difficult, if not impossible, to detect but in the intermediate and late phases of the development, when the tumors become diagnosable, their dynamics is Gompertzian.

\section{References}

[1] A.K. Laird, Br. J. Cancer 18, 490 (1964).

[2] M. Marušić, Ž. Bajzer, J.P. Freyer, S. Vuk-Pavlović, Cell Prolif. 21, 13 (1994).

[3] I. Bozic, T. Antal, H. Ohtsuki et al., Proc. Natl. Acad. Sci. 107, 18545 (2010).

[4] M.P. Little, J.H. Hendry, PLoS Comput Biol. 13, e1005391 (2017).

[5] C. Tomasetti, B. Vogelstein, G. Parmigiani, Proc. Natl. Acad. Sci. 110, 2001 (2013).

[6] U. Schneider, Med. Phys. 36, 1138 (2009).

[7] I. Shuryak, P. Hahnfeldt, L. Hlatky, R.K. Sachs, D.J. Brenner, Radiat. Environ. Biophys. 48, 263 (2009).

[8] R. Khordad, H.R. Rastegar Sedehi, Eur. Phys. J. Plus 131, 291 (2016).

[9] J. Tang, W. Georgescu, T. Deschamps, S.M. Yannone, S.V. Costes, in: Genomic Instability and Cancer Metastasis: Mechanisms, Emerging Themes, and Novel Therapeutic Strategies, Eds. Ch. Maxwell, C. Roskelley, Springer, 2014, Ch. 4.

[10] B.R. Scott, D.M. Walker, Y. Tesfaigzi, H. Schöllnberger, V. Walker, Nonlinear. Biol. Toxicol. Med. 1, 93 (2003).

[11] Y. Hattori, A. Yokoya, R. Watanabe, BMC Systems Biol. 9, 90 (2015).

[12] M. Pollycove, L.E. Feinendegen, Human Exp. Toxicol. 22, 290 (2003).

[13] E.I. Azzam, J.P. Jay-Gerin, D. Pain, Cancer Lett. 327, 48 (2011).

[14] L. Dobrzyński, K.W. Fornalski, J. Reszczyńska, M.K. Janiak, Dose-Response 17, 1 (2019).
[15] C.M. Fleck, H. Schöllnberger, M.M. Kottbauer, T. Dockal, U. Prufert, Math. Biosci. 155, 13 (1999).

[16] A.M. Kellerer, H.H. Rossi, Radiat. Res. 75, 471 (1978).

[17] L. Dobrzyński, K.W. Fornalski, Y. Socol, J. Reszczyńska, Radiat. Res. 186, 396 (2016).

[18] B. Alberts, A. Johnson, J. Lewis, M. Raff, K. Roberts, P. Walter, Molecular Biology of the Cell, 4th ed., Garland Sci., New York 2002.

[19] M.H. Bailey, C. Tokheim, E. Porta-Pardo et al., Cell 173, 371 (2018).

[20] S. Moolgavkar, J. Nat. Cancer Inst. 61, 49 (1978).

[21] B. Vogelstein, N. Papadopoulos, V.E. Velculescu, S. Zhou, L.A. Diaz Jr., K.W. Kinzler, Science 339, 1546 (2013).

[22] M.J. Renan, Mol. Carcinogenesis 7, 139 (1993).

[23] W.C. Hahn, C.M. Counter, A.S. Lundberg, R.L. Beijersbergen, M.W. Brooks, R.A. Weinberg, Nature 400, 464 (1999).

[24] W.C. Hahn, R.A. Weinberg, N. Engl. J. Med. 347, 1593 (2002).

[25] M. Andersson, H.H. Storm, J. Natl. Cancer Inst. 84, 1318 (1992).

[26] K.W. Fornalski, L. Dobrzyński, M.K. Janiak, Dose-Response 9, 477 (2011).

[27] K.W. Fornalski, Int. J. Low Radiat. 9, 370 (2014).

[28] P. Wysocki, M.Sc. Thesis, Faculty of Physics, Warsaw University of Technology, 2019.

[29] K.P. Chatzipapas, P. Papadimitroulas, D. Emfietzoglou, S.A. Kalospyros, M. Hada, A.G. Georgakilas, G.C. Kagadis, Cancers (Basel) 12, E799 (2020).

[30] M.Y. Tsai, Z. Tian, N. Qin, C. Yan, Y. Lai, S.H. Hung, Y. Chi, X. Jia, Med. Phys. 47, 1958 (2020).

[31] P. Armitage, R. Doll, Br. J. Cancer 8, 1 (1954).

[32] M.M. Gonzalez, J.A. González Joa, L.E. Bergues Cabrales et al., BMC Cancer 17, 174 (2017). 